\title{
Longitudinal changes in pancreatic and adipocyte hormones following Roux-en-Y gastric bypass surgery
}

\author{
M. M. Swarbrick • K. L. Stanhope • \\ I. T. Austrheim-Smith • M. D. Van Loan • M. R. Ali • \\ B. M. Wolfe • P. J. Havel
}

Received: 26 March 2008 / Accepted: 14 July 2008 / Published online: 15 August 2008

(C) Springer-Verlag 2008

\begin{abstract}
Aims/hypothesis Bariatric surgery is an effective treatment for severe obesity, as in addition to dramatic weight loss, co-morbidities such as type 2 diabetes are frequently resolved. Although altered gastrointestinal peptide hormone secretion and its relationship with post-surgical improvements in insulin sensitivity has been studied, much less is known about long-term changes in pancreatic and adipose tissue-derived hormones. Our objective was to conduct a comprehensive longitudinal investigation of the endocrine
\end{abstract}

M. M. Swarbrick $\cdot$ K. L. Stanhope $\cdot$ P. J. Havel $(\bowtie)$

Department of Molecular Biosciences, School of Veterinary

Medicine, University of California, Davis,

One Shields Ave,

Davis, CA 95616-5270, USA

e-mail: pjhavel@ucdavis.edu

M. M. Swarbrick • K. L. Stanhope · M. D. Van Loan • P. J. Havel

Department of Nutrition,

College of Agricultural and Environmental Sciences,

University of California,

Davis, CA, USA

I. T. Austrheim-Smith • M. R. Ali

Department of Surgery, School of Medicine,

University of California,

Davis, CA, USA

M. D. Van Loan

United States Department of Agriculture,

Agricultural Research Service,

Western Human Nutrition Research Center,

Davis, CA, USA

B. M. Wolfe

Department of Surgery, Oregon Health \& Science University,

Portland, OR, USA changes following Roux-en-Y gastric bypass surgery (RYGBP), focusing on pancreatic and adipocyte hormones and systemic markers of inflammation.

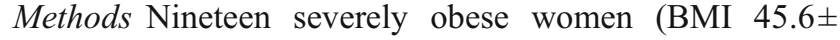
$1.6 \mathrm{~kg} / \mathrm{m}^{2}$ ) were studied prior to RYGBP, and at $1,3,6$, and 12 months after RYGBP. Body composition was assessed before surgery and at 1 and 12 months.

Results Pre-surgical adiposity was correlated with circulating adipocyte hormones (leptin, visfatin) and inflammatory molecules (IL-6, high sensitivity C-reactive protein [hsCRP], monocyte chemoattractant protein-1). As expected, RYGBP reduced fat mass and fasting insulin and glucose concentrations. In addition, reductions of fasting pancreatic polypeptide (PP) and glucagon concentrations were observed at 1 and 3 months, respectively. In the 12 months following RYGBP, concentrations of most adipocyte hormones (leptin, acylation-stimulating hormone and visfatin, but not retinol-binding hormone-4) and inflammatory molecules (IL-6, hsCRP and soluble intracellular adhesion molecule-1) were significantly reduced. Reductions of insulin resistance (measured by homeostasis model assessment of insulin resistance) were independently associated with changes of glucagon, visfatin and PP. Presurgical HMW adiponectin concentrations independently predicted losses of body weight and fat mass.

Conclusions/interpretation These results suggest that pancreatic and adipocyte hormones may contribute to the longterm resolution of insulin resistance after RYGBP.

Keywords Clinical science · Cytokines - Gastro-entero pancreatic factors $\cdot$ Human $\cdot$ Insulin sensitivity and resistance - Other hormones - Other islet cells/hormones . Weight regulation and obesity 


$\begin{array}{ll}\text { Abbreviations } \\ \text { ASP } & \text { acylation-stimulating protein } \\ \text { CRP } & \text { C-reactive protein } \\ \text { GLP-1 } & \text { glucagon-like peptide 1 } \\ \text { HMW } & \text { high molecular weight } \\ \text { HOMA- } & \text { homeostasis model assessment of insulin re- } \\ \text { IR } & \text { sistance } \\ \text { hSCRP } & \text { high-sensitivity C-reactive protein } \\ \text { MCP-1 } & \text { monocyte chemoattractant protein-1 } \\ \text { PP } & \text { polypeptide } \\ \text { RBP4 } & \text { retinol-binding protein-4 } \\ \text { RYGBP } & \text { Roux-en-Y gastric bypass } \\ \text { sICAM-1 } & \text { soluble intercellular adhesion molecule-1 }\end{array}$

\section{Introduction}

Bariatric surgery is the most effective method for producing weight loss in patients with severe obesity (BMI $>40 \mathrm{~kg} /$ $\mathrm{m}^{2}$ ). In addition to weight loss, the co-morbidities of severe obesity, particularly type 2 diabetes mellitus, insulin resistance, hyperlipidaemia, sleep apnoea and hypertension are frequently resolved following surgery [1]. Accordingly, the number of bariatric surgical procedures performed in the USA increased eightfold between 1998 and 2004 [2]. Similar increases have been reported in many other countries [3].

There is considerable interest in the physiological mechanisms by which bariatric surgery, in particular Roux-en-Y gastric bypass (RYGBP), resolves the comorbidities of severe obesity, as understanding them could lead to the development of new (non-surgical) therapeutic strategies. Much of the research to date has focused on gastrointestinal hormones, such as ghrelin, glucagon-like peptide-1 (GLP-1) and peptide-YY, and their relationships with the weight loss, reduction of appetite and improved insulin sensitivity that follow RYGBP [4]. However, concomitant changes in pancreatic (glucagon, pancreatic polypeptide [PP] and amylin) and adipocyte-derived hormones have not been as intensively investigated.

Over the last two decades, adipose tissue has been recognised as an active source of hormones involved in energy homeostasis and substrate metabolism [5]. Adipocyte-derived hormones include leptin, adiponectin and acylation-stimulating protein (ASP), as well as the more recently identified visfatin (also known as PBEF/NAMPT) [6]. With the exception of adiponectin, circulating concentrations of these hormones are usually increased in obesity and insulin-resistant states, and decline during weight loss [7]. A role for adipocyte-derived retinol-binding protein-4 (RBP4) in systemic insulin resistance has also been recently proposed: in transgenic mice, adipose-specific GLUT4 knockout increased serum RBP4 concentrations, which in turn led to insulin resistance by impairing skeletal muscle insulin action and increasing hepatic glucose output [8].

Obesity is also characterised by the infiltration of macrophages into adipose tissue, promoting a state of chronic, low-grade inflammation [9]. Relative to lean, healthy individuals, obesity is associated with elevated concentrations of C-reactive protein (CRP) [10], IL-6 [11], monocyte chemoattractant protein-1 (MCP-1) [12] and soluble intercellular adhesion molecule-1 (sICAM-1) [13]. This inflammatory state is implicated in the development of many complications of severe obesity, in particular, atherosclerosis, insulin resistance and type 2 diabetes [14].

The objective of this study was to conduct a comprehensive longitudinal examination of the endocrine and metabolic changes following RYGBP, focusing on pancreatic and adipocyte hormones as well as inflammatory markers. Many previous studies of the endocrine effects of bariatric surgery have either been cross-sectional in design or have only examined a single time-point after surgery. We have previously reported that concentrations of high molecular weight (HMW) adiponectin increase following RYGBP, and that these increases were proportional to relative improvements in insulin resistance [15]. We now provide additional information pertaining to other adipocyte-derived hormones (leptin, ASP, visfatin, RBP4), inflammatory markers (IL-6, high-sensitivity CRP [hsCRP]) and pancreatic hormones (proinsulin, glucagon, PP and amylin) after RYGBP in these severely obese individuals.

\section{Methods}

Subjects Nineteen severely obese women (aged 40.6 1.8 years, mean $\pm \mathrm{SEM}$ ) underwent RYGBP surgery performed at the University of California, Davis Medical Center, or at Mercy San Juan Hospital in Sacramento, CA, USA. Although patients were encouraged to lose weight prior to surgery, their weight had been stable for the preceding 3 months. Fasting blood samples were collected prior to surgery, and at $1,3,6$ and 12 months postoperatively. Body composition was assessed prior to surgery and at 1 and 12 months. The experimental protocol was approved by Institutional Review Board of UC Davis, and all participants provided written informed consent for participation in the study.

Anthropometric and biochemical measurements Weight, height, and waist and hip circumferences were measured using standard methods. Body composition was determined by air-displacement plethysmography (BodPod, Life Measurements, Concord, CA, USA). 
All biochemical measurements were performed on fasting plasma samples, except measurements of RBP4 and visfatin, which were performed on fasting serum samples. Insulin, proinsulin, leptin, glucagon and cortisol were measured by radioimmunoassay (Linco, St Charles, MO, USA). Plasma glucose was measured with a YSI glucose analyser (Yellow Springs, OH, USA). Homeostatic model assessment of insulin resistance (HOMA-IR) was calculated as previously described [16]. NEFA concentrations were measured with an enzymatic assay (Waco Chemicals, Richmond, VA, USA). RBP4 was measured by ELISA (Alpco, Salem, NH, USA). MCP-1 and sICAM-1 were measured by ELISA, and IL-6 was measured using a Quantikine HS ELISA (all from R\&D Systems, Minneapolis, MN, USA). ASP and amylin were measured by ELISA (Linco). The amylin assay measured human amylin, deaminated amylin, the 1-20 fragment of amylin, but not reduced amylin. PP was measured by radioimmunoassay (Alpco). CRP was measured with an Immulite analyser and hsCRP reagents (Diagnostic Products, Los Angeles, CA, USA). Visfatin was measured by ELISA (Adipogen, Seoul, South Korea).

Statistical analysis Statistical analysis was performed using GraphPad Prism v. 4 (San Diego, CA, USA) and JMP Start Statistics (SAS, Cary, NC, USA). All continuous variables were assessed for normality using the Kolmogorov-Smirnov test. Comparisons between baseline and post-surgical values were made with one-way repeated measures ANOVA or the non-parametric Friedman test. Post-test comparisons were made with Bonferroni's multiple comparison test, with the pre-surgical values used as the reference. For SICAM-1 and
MCP-1, comparisons between baseline and 12 months were made using paired $t$ tests. Relationships between continuous variables were assessed by non-parametric Spearman rank correlation. Multivariate analyses were performed using forward stepwise multiple logistic regression, as described earlier [15]. The two-sided level of significance was $p<$ 0.05 . All mean values are shown \pm SEM.

\section{Results}

Baseline characteristics and changes of body weight and body composition following RYGBP Body composition and biochemical measurements in the 19 participants are shown in Table 1. At 1, 3, 6 and 12 months after RYGBP, average reductions in body weight were $10.3 \pm 0.4 \%, 17.8 \pm 1.0 \%$, $24.7 \pm 1.0 \%$ and $31.9 \pm 1.5 \%$, respectively. Weight loss was predominantly due to changes in fat mass, which was reduced by $11.7 \pm 0.8 \%(7.6 \pm 0.5 \mathrm{~kg})$ at 1 month and $49.6 \pm$ $2.5 \%(32.7 \pm 2.4 \mathrm{~kg})$ at 12 months. This was accompanied by a modest, but significant, decrease of fat-free mass: $8.7 \pm$ $0.9 \%(5.4 \pm 0.4 \mathrm{~kg})$ at 1 month and $15.2 \pm 2.9 \%(9.2 \pm 1.7 \mathrm{~kg})$ at 12 months. Waist circumference and WHR were significantly reduced at 1 and 6 months, respectively.

Plasma glucose, HOMA-IR and pancreatic hormones Three individuals presented with fasting hyperglycaemia (plasma glucose $\geq 7.0 \mathrm{mmol} / \mathrm{l}$ ) and another three with impaired fasting glucose $(6.1-6.9 \mathrm{mmol} / \mathrm{l})$ prior to surgery. After RYGBP, plasma glucose and insulin concentrations decreased progressively over the next 12 months (Fig. 1a,b).

Table 1 Changes of body composition, hormones and markers of inflammation in severely obese women following RYGBP

\begin{tabular}{|c|c|c|c|c|c|c|}
\hline Variable & Pre-operative & 1 month & 3 months & 6 months & 12 months & Overall $p$ value \\
\hline$n$ & 19 & 19 & 18 & 19 & 19 & - \\
\hline Body weight (kg) & $126.4 \pm 3.6$ & $113.4 \pm 3.3^{* * *}$ & $107.2 \pm 4.4^{* * *}$ & $95.4 \pm 3.3 * * *$ & $85.9 \pm 2.8 * * *$ & $<0.0001$ \\
\hline BMI $\left(\mathrm{kg} / \mathrm{m}^{2}\right)$ & $45.6 \pm 1.6$ & $41.0 \pm 1.5^{* * *}$ & $38.8 \pm 2.1 * * *$ & $34.5 \pm 1.5^{* * *}$ & $30.8 \pm 1.0 * * *$ & $<0.0001$ \\
\hline Fat mass $(\mathrm{kg})$ & $65.5 \pm 2.7$ & $58.2 \pm 2.5 * *$ & - & - & $32.9 \pm 1.8 * * *$ & $<0.0001$ \\
\hline Fat-free mass $(\mathrm{kg})$ & $60.9 \pm 2.0$ & $55.2 \pm 1.6^{* * *}$ & - & - & $53.0 \pm 2.5^{* * *}$ & $<0.0001^{\mathrm{a}}$ \\
\hline Waist circumference $(\mathrm{cm})$ & $130 \pm 2$ & $119 \pm 3 *$ & $111 \pm 3 * * *$ & $104 \pm 2 * * *$ & $100 \pm 2 * * *$ & $<0.0001$ \\
\hline WHR & $0.95 \pm 0.03$ & $0.90 \pm 0.02$ & $0.89 \pm 0.02$ & $0.87 \pm 0.02 *$ & $0.88 \pm 0.02 * *$ & 0.046 \\
\hline HOMA-IR & $7.4 \pm 1.0$ & $4.0 \pm 0.5 * * *$ & $3.1 \pm 0.3 * * *$ & $2.6 \pm 0.2 * * *$ & $2.3 \pm 0.2 * * *$ & $<0.0001$ \\
\hline Proinsulin:insulin ratio (\%) & $16.7 \pm 1.6$ & $15.6 \pm 1.7$ & $16.4 \pm 1.3$ & $16.2 \pm 1.3$ & $15.1 \pm 1.1$ & 0.59 \\
\hline NEFA $(\mathrm{mmol} / \mathrm{l})$ & $0.28 \pm 0.03$ & $0.37 \pm 0.04$ & $0.26 \pm 0.03$ & $0.23 \pm 0.04$ & $0.20 \pm 0.02$ & $<0.0001$ \\
\hline Cortisol (nmol/l) & $232 \pm 22$ & $265 \pm 28$ & $262 \pm 25$ & $295 \pm 30$ & $257 \pm 25$ & $0.10^{\mathrm{a}}$ \\
\hline IL-6 (pg/ml) & $4.16 \pm 0.50$ & $3.88 \pm 0.33$ & - & - & $2.11 \pm 0.20 * * *$ & $<0.0001^{\mathrm{a}}$ \\
\hline hsCRP (mg/l) & $12.6 \pm 2.1$ & $9.5 \pm 1.4$ & $7.4 \pm 2.0^{* *}$ & $5.2 \pm 1.4^{* * *}$ & $2.8 \pm 0.7 * * *$ & $<0.0001$ \\
\hline sICAM-1 (ng/ml) & $243 \pm 9$ & - & - & - & $216 \pm 9 *$ & 0.011 \\
\hline MCP-1 (pg/ml) & $163 \pm 8$ & - & - & - & $161 \pm 8$ & 0.81 \\
\hline
\end{tabular}

Data are shown as mean \pm SEM. Comparisons between baseline and each time-point were made using repeated measures one-way ANOVA, except where its non-parametric equivalent, the Friedman test was used ${ }^{a}$. Levels of significance at each time-point were calculated using Bonferroni's multiple comparison test.

$* p<0.05, * * p<0.01, * * * p<0.0001$ 
a

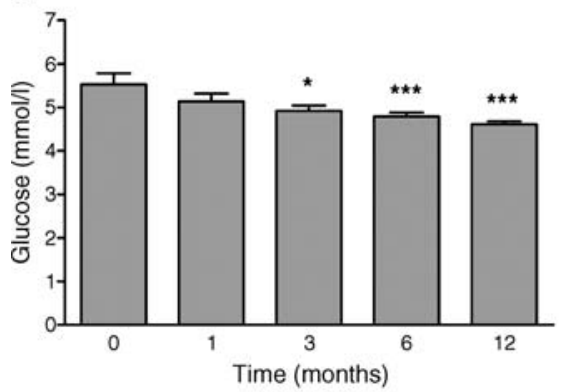

d

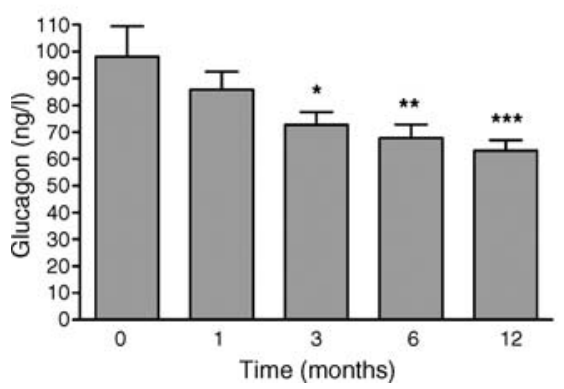

b

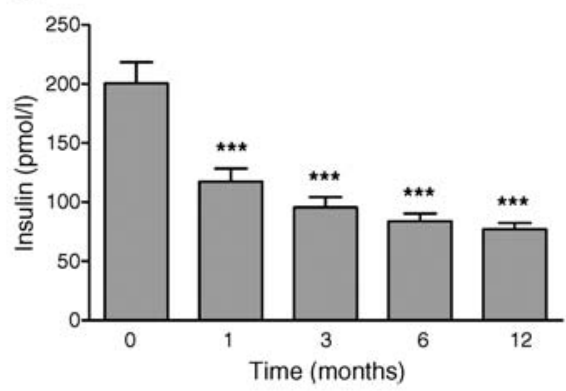

e

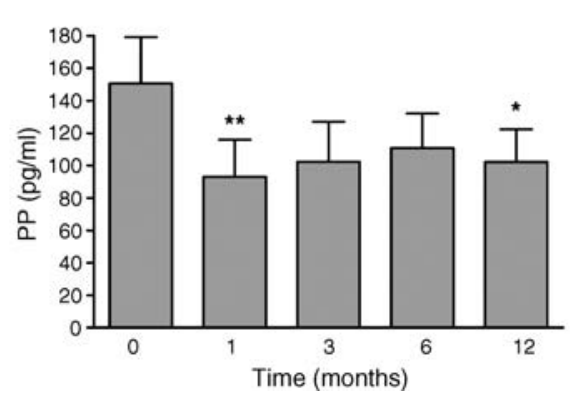

C

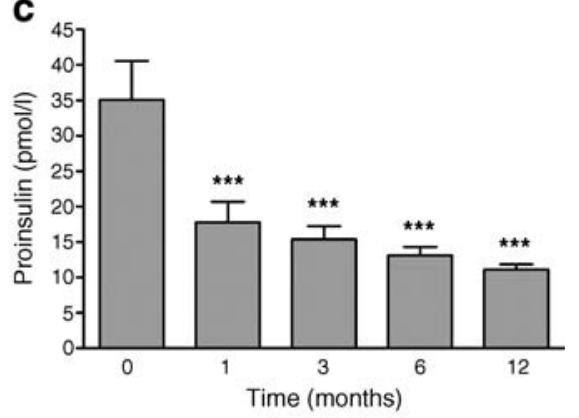

f

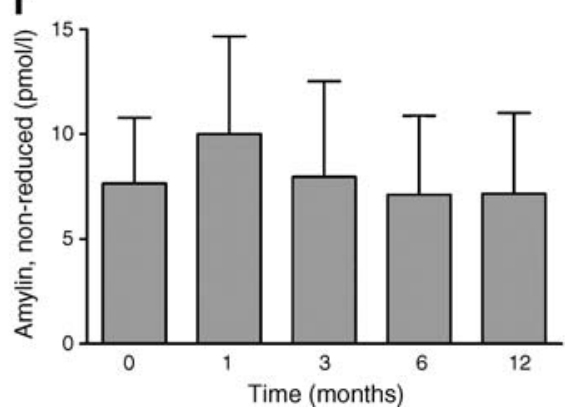

Fig. 1 Fasting plasma concentrations of glucose, insulin and other pancreatic hormones following RYGBP. Differences between time-points were determined by repeated measures one-way ANOVA. ${ }^{*} p<0.05$, $* * p<0.01$ and $* * * p<0.0001$ relative to pre-surgical values
After 1 month, fasting insulin concentrations had decreased by $34 \pm 8 \%$, and glucose levels were normalised in all individuals. Accordingly, HOMA-IR was significantly reduced by 1 month and continued to decrease thereafter (Table 1). Plasma proinsulin concentrations decreased in parallel with changes of insulin (Fig. 1c), such that the proinsulin/insulin ratio (a marker of pancreatic beta cell dysfunction [17]) was unchanged following RYGBP (Table 1). When stratified by the presence or absence of presurgical hyperglycaemia, the proinsulin/insulin ratio did not change significantly in either group (data not shown). This contrasts with the reported improvement of dynamic beta cell function (accompanied by a reduced proinsulin/insulin ratio) in patients with weight loss induced by vertical gastric banding and jejunoileal bypass [18].

Fasting glucagon, PP and amylin concentrations were also measured. In these severely obese women, fasting plasma glucagon concentrations at baseline $(98 \pm 11 \mathrm{ng} / \mathrm{l})$ were twice as high as those we have previously reported in normal weight and overweight women, using the same assay $[19,20]$. Glucagon concentrations decreased following RYGBP, being reduced by $22 \pm 8 \%$ at 3 months, $26 \pm 5 \%$ at 6 and, $31 \pm 4 \%$ at 12 months (Fig. 1d). Plasma PP concentrations were significantly decreased at $1(-27 \pm$ $10 \%)$ and 12 months $(-17 \pm 13 \%)$, but not at 3 or 6 months (Fig. 1e). Amylin concentrations did not change after RYGBP (Fig. 1f).
Adipocyte hormones and inflammatory molecules As expected, leptin concentrations prior to surgery were proportional to body weight, BMI and fat mass (data not shown). Concentrations of other adipocyte hormones and inflammatory markers (visfatin, IL-6, MCP-1 and hsCRP) were also correlated with body adiposity, but not with fasting insulin concentrations or HOMA-IR (Table 2). Visfatin concentrations were also positively correlated with fat-free mass. Concentrations of RBP4, ASP and sICAM-1 were not significantly associated with measures of body composition or insulin resistance at baseline.

Concentrations of most adipocyte hormones decreased significantly 1 month after RYGBP (Fig. 2). Reductions of leptin $(-36 \pm 3 \%)$, visfatin $(-20 \pm 6 \%)$, RBP4 $(-28 \pm 6 \%)$ and ASP $(-18 \pm 7 \%)$ were observed. At 12 months, similarly, concentrations of leptin and visfatin were reduced by $64 \pm$ $4 \%$ and $41 \pm 8 \%$, respectively. However, concentrations of ASP and RBP4 at twelve months were not statistically different from pre-surgical values.

RYGBP also led to a progressive reduction of some, but not all markers of inflammation (Table 1). IL-6 concentrations were unchanged at 1 month after RYGBP, but were reduced by $43 \pm 7 \%$ at 12 months $(p<0.001)$. hsCRP concentrations were reduced by $46 \pm 7 \%$ and $74 \pm 7 \%$ at 3 and 12 months, respectively. Concentrations of sICAM-1 were reduced by $10 \pm 4 \%$ after 12 months, while MCP-1 concentrations did not change significantly (Table 1). 
Table 2 Significant correlations between continuous variables at baseline and at 12 months

\begin{tabular}{|c|c|c|c|}
\hline Variable 1 & Variable 2 & $r$ & $p$ value \\
\hline \multicolumn{4}{|c|}{ Significant correlations at baseline } \\
\hline \multirow[t]{3}{*}{ Visfatin } & Body weight & 0.65 & 0.0028 \\
\hline & Waist circumference & 0.50 & 0.035 \\
\hline & Fat-free mass & 0.55 & 0.015 \\
\hline \multirow[t]{3}{*}{ IL-6 } & Body weight & 0.59 & 0.007 \\
\hline & BMI & 0.67 & 0.002 \\
\hline & Fat mass & 0.70 & 0.0009 \\
\hline \multirow[t]{3}{*}{ hsCRP } & Body weight & 0.55 & 0.015 \\
\hline & Waist circumference & 0.51 & 0.030 \\
\hline & Fat mass & 0.55 & 0.014 \\
\hline MCP-1 & BMI & 0.60 & 0.007 \\
\hline \multicolumn{4}{|c|}{ Significant correlations at 12 month follow-up } \\
\hline \multirow[t]{3}{*}{ IL-6 } & WHR & 0.48 & 0.036 \\
\hline & Insulin & 0.67 & 0.0017 \\
\hline & HOMA-IR & 0.59 & 0.0074 \\
\hline sICAM-1 & Glucose & -0.48 & 0.036 \\
\hline
\end{tabular}

Before RYGBP and at 12 month follow-up, hormones and inflammatory markers were tested for correlation with measures of body composition (weight, BMI, fat mass, lean mass, waist circumference, WHR), fasting glucose and insulin concentrations, and insulin resistance (HOMA-IR) by non-parametric Spearman rank. The level of significance was $p<0.05$

At 12 months, the relationship between leptin concentrations and adiposity remained significant (data not shown), while leptin concentrations were also positively correlated with fasting insulin $(r=0.55, p=0.015)$ and HOMA-IR $(r=0.48, p=0.039)$. IL-6 concentrations were positively correlated with WHR, fasting insulin and HOMA-IR, and SICAM-1 concentrations were negatively correlated with fasting glucose.

Hormones independently associated with changes of body weight and body composition In our previous report [15], we found that changes of HMW adiponectin were associated with the changes in fat mass ( $\Delta$ fat mass) in the 12 months following RYGBP, independently of age and initial BMI. Using this model, we found that $\Delta$ fat mass was also related to changes of leptin (model 1, Table 3). This model accounted for $71 \%$ of the variance in $\Delta$ fat mass.

We next tested which baseline variables were predictive of $\Delta$ fat mass. Of the hormones studied, only baseline HMW adiponectin concentrations predicted $\Delta$ fat mass, independently of age and BMI at baseline (model 2, Table 3 and Fig. 3a). Similar results were obtained when we examined proportional changes of fat mass; however, the models explained less of the overall variance (data not shown).

Variables related to the change of body weight over the 12 months ( $\Delta$ body weight) were also studied. As for $\Delta$ fat mass, changes of both leptin and HMW adiponectin were
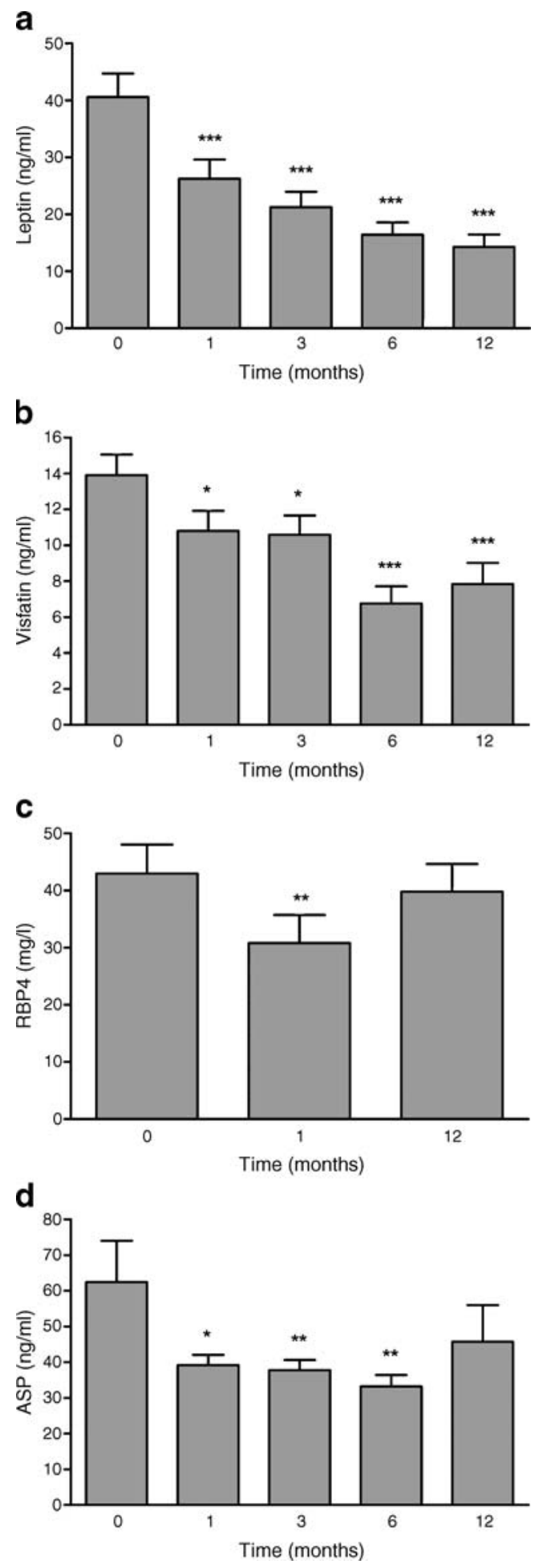

Fig. 2 Fasting plasma concentrations of adipocyte hormones following RYGBP. Differences between time-points were determined by repeated measures one-way ANOVA. ${ }^{*} p<0.05,{ }^{* *} p<0.01$, and $* * * p<0.0001$ relative to pre-surgical values 
Table 3 Multivariate analyses of variables associated with RYGBP-induced changes of body weight, fat mass, fasting insulin and HOMA-IR

\begin{tabular}{|c|c|c|c|c|c|c|c|}
\hline Model & $n$ & Dependent variable & Independent variable & $\beta$ coefficient & SE & $p$ value & Model $r^{2}$ (adj) \\
\hline \multirow[t]{4}{*}{1} & \multirow[t]{4}{*}{19} & $\Delta$ Fat mass $(\mathrm{kg})$ & Age & 0.432 & 0.194 & 0.043 & \multirow[t]{4}{*}{0.71} \\
\hline & & \multirow[t]{3}{*}{$0-12$ months } & Initial BMI & -0.237 & 0.222 & 0.30 & \\
\hline & & & $\Delta$ Leptin & 0.349 & 0.111 & 0.0070 & \\
\hline & & & $\Delta \mathrm{HMW}$ Ad & -3.786 & 1.217 & 0.0077 & \\
\hline \multirow[t]{3}{*}{2} & \multirow[t]{3}{*}{19} & $\Delta$ Fat mass $(\mathrm{kg})$ & Age & 0.287 & 0.219 & 0.21 & \multirow[t]{3}{*}{0.49} \\
\hline & & \multirow[t]{2}{*}{$0-12$ months } & Initial BMI & -0.728 & 0.246 & 0.0098 & \\
\hline & & & Baseline HMW Ad & -4.695 & 1.721 & 0.016 & \\
\hline \multirow[t]{3}{*}{3} & \multirow[t]{3}{*}{19} & $\Delta$ Weight $(\mathrm{kg})$ & Age & 0.357 & 0.235 & 0.15 & \multirow[t]{3}{*}{0.64} \\
\hline & & \multirow[t]{2}{*}{$0-12$ months } & $\Delta$ Leptin & 0.478 & 0.120 & 0.0012 & \\
\hline & & & $\Delta \mathrm{HMW}$ Ad & -3.740 & 1.459 & 0.022 & \\
\hline \multirow[t]{4}{*}{4} & \multirow[t]{4}{*}{19} & $\Delta$ Weight $(\mathrm{kg})$ & Age & -0.070 & 0.246 & 0.78 & \multirow[t]{4}{*}{0.58} \\
\hline & & \multirow[t]{3}{*}{$0-12$ months } & Baseline HMW Ad & -7.604 & 1.958 & 0.0017 & \\
\hline & & & Baseline hsCRP & -0.628 & 0.229 & 0.016 & \\
\hline & & & Baseline ghrelin & 0.082 & 0.038 & 0.047 & \\
\hline \multirow[t]{4}{*}{5} & \multirow[t]{4}{*}{19} & $\Delta$ Fasting insulin & Age & 0.601 & 1.789 & 0.74 & \multirow[t]{4}{*}{0.47} \\
\hline & & \multirow[t]{3}{*}{$0-12$ months } & $\Delta$ Visfatin & 7.429 & 2.348 & 0.0069 & \\
\hline & & & $\Delta$ Glucagon & 0.846 & 0.325 & 0.021 & \\
\hline & & & $\Delta \mathrm{PP}$ & 2.273 & 0.938 & 0.030 & \\
\hline \multirow[t]{3}{*}{6} & \multirow[t]{3}{*}{18} & $\Delta$ HOMA-IR & Age & -0.187 & 0.092 & 0.062 & \multirow[t]{3}{*}{0.42} \\
\hline & & \multirow[t]{2}{*}{ 0-12 months } & $\Delta$ Glucagon & 0.072 & 0.020 & 0.0031 & \\
\hline & & & $\Delta$ Visfatin & 0.343 & 0.147 & 0.035 & \\
\hline \multirow[t]{4}{*}{7} & \multirow[t]{4}{*}{18} & $\% \Delta$ HOMA-IR & Age & 0.732 & 0.321 & 0.040 & \multirow[t]{4}{*}{0.59} \\
\hline & & \multirow[t]{3}{*}{ 0-12 months } & $\Delta \mathrm{HMW}$ Ad & -8.995 & 2.511 & 0.0033 & \\
\hline & & & $\triangle \mathrm{NEFA}$ & -76.77 & 21.547 & 0.0035 & \\
\hline & & & $\Delta \mathrm{IL}-6$ & -2.391 & 1.139 & 0.056 & \\
\hline
\end{tabular}

Stepwise multiple logistic regression was performed using the least squares method, as described by Swarbrick et al. [15].

Ad, adiponectin; $r^{2}$ (adj), adjusted proportion of the variance accounted for by the model

independently associated with $\Delta$ body weight (model 3 , Table 3). $\Delta$ Body weight was also independently predicted by baseline concentrations of HMW adiponectin (Fig. 3b), hsCRP (Fig. 3c) and ghrelin (model 4, Table 3).

Changes of each of the hormones measured here were also tested for association with the changes of fat-free mass after surgery. None of these were correlated with $\Delta$ fat-free mass (data not shown).

Hormones independently associated with changes of fasting insulin and HOMA-IR In all participants, changes of fasting insulin concentrations between baseline and 12 months $(\Delta$ insulin) were significantly and independently associated with changes of visfatin $(p=0.0069)$ (Fig. 3d), glucagon $(p=0.021)$ and PP $(p=0.030$; model 5 , Table 3$)$. This model accounted for $47 \%$ of the variation in $\Delta$ insulin. Due to the non-linear relationship between $\Delta$ glucagon and $\Delta$ insulin, the proportional (\%) changes were examined and these were significantly correlated ( $r=0.56, p=0.013$; Fig. 3e).

Relationships between the changes of hormones and changes of HOMA-IR over the 12 months were also examined. Analyses were limited to participants in whom HOMA-IR improved by $>15 \%$ at 12 months after surgery [15], which yielded 18 participants. As for $\Delta$ insulin, the absolute changes of HOMA-IR over the 12 months were associated with changes of glucagon and visfatin (model 6, Table 3).

The final model (model 7, Table 3) examined relative changes of HOMA-IR, to account for the substantial absolute improvement of insulin sensitivity that occurred in the patients with hyperglycaemia at baseline. As previously described [15], the proportional improvement in HOMA-IR (\% $\%$ HOMA-IR) over the 12 months was related to age, $\triangle$ NEFA (Fig. 3f) and $\triangle$ HMW adiponectin (model 7, Table 3). In this model, the change of IL-6 concentrations also tended to be independently associated with $\% \Delta$ HOMA-IR $(p=0.056$; Fig. $3 \mathrm{~g})$. This model explained $59 \%$ of the variation in $\% \Delta$ HOMA-IR.

\section{Discussion}

This study investigated the longitudinal changes of pancreatic and adipocyte hormones following RYGBP in severely obese women. Markers of inflammation were also examined. While previous studies have reported changes of several of these variables following bariatric surgery, we believe that this study is the first longitudinal 
a
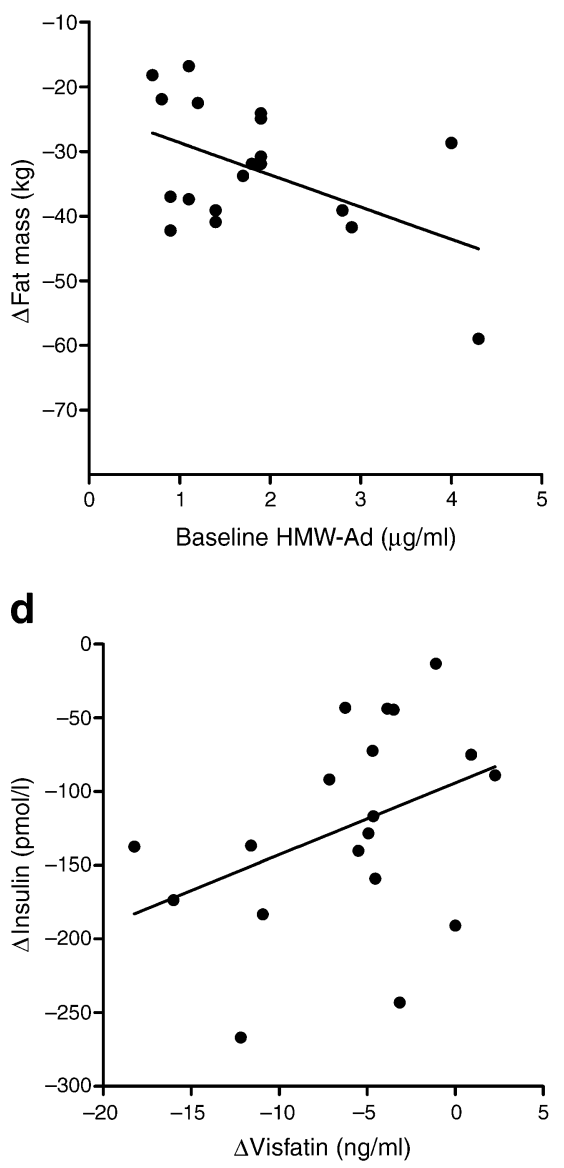

b

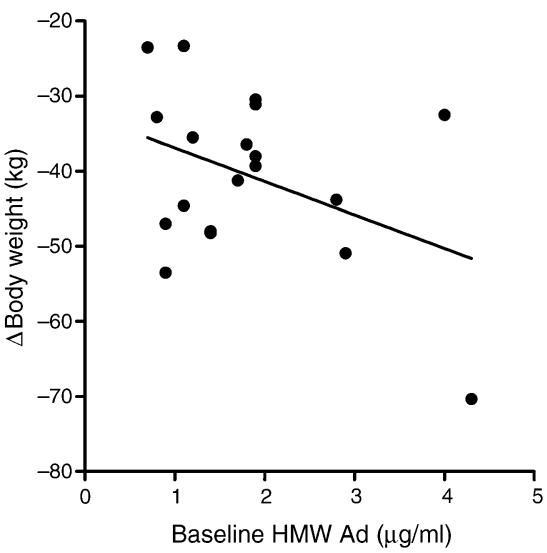

e

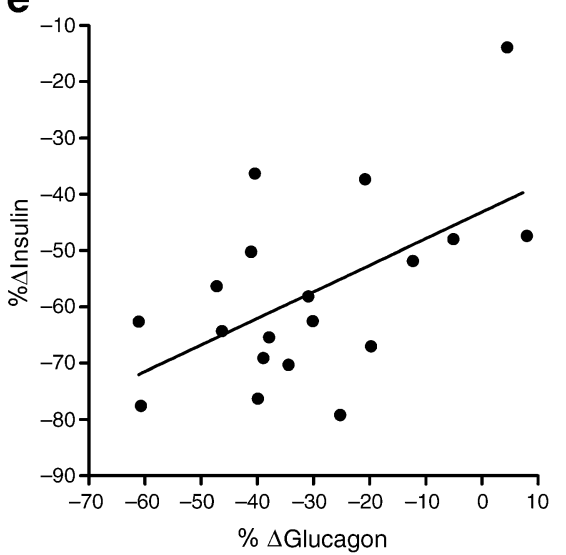

C

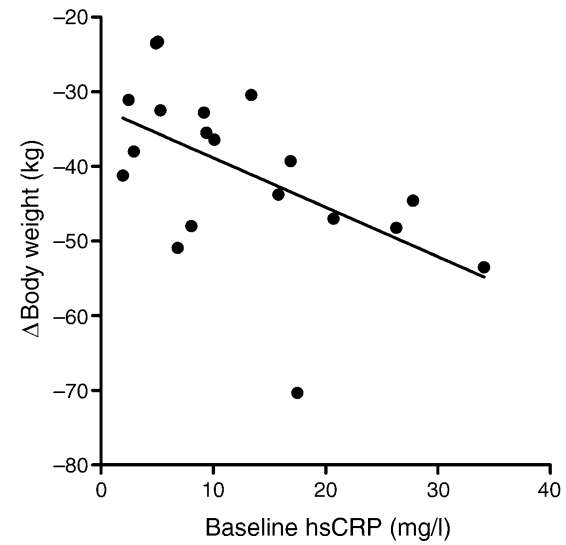

f

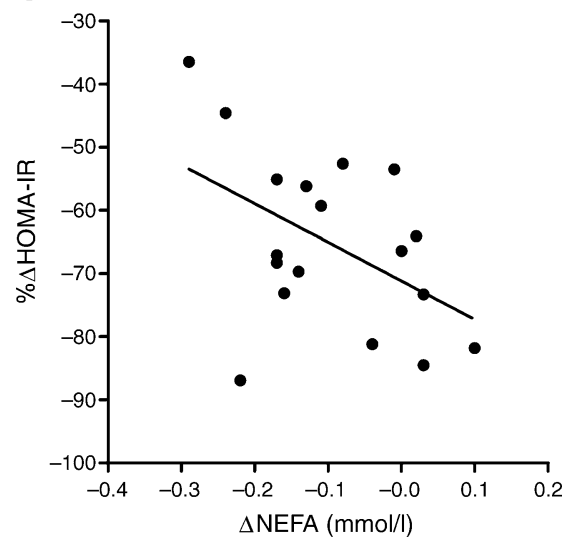

g

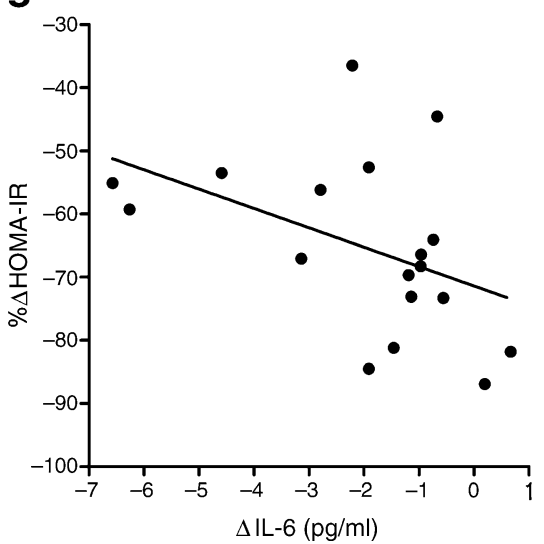

Fig. 3 Significant independent predictors of absolute and proportional changes of body weight, fat mass, fasting insulin concentrations and HOMA-IR. a Baseline HMW adiponectin (Ad) concentrations predicted $\Delta$ fat mass, $r=-0.50, p=0.016$ (model 2, Table 3 ) and b $\Delta$ body weight, $r=-0.40, p=0.0017$ (model 4). c Baseline hsCRP concentrations were also associated with $\Delta$ body weight following

examination to be comprehensive in scope, due to the number of measurements performed and the frequency of follow-up examinations. A further strength of the present study was that changes of body composition were assessed. Its main limitations were that the sample size
RYGBP, $r=-0.55, p=0.016$ (model 4$)$. $\mathbf{d} \Delta$ Visfatin and $\Delta$ insulin were significantly correlated, $r=0.39, p=0.0069$ (model 5). e Proportional changes of glucagon and insulin were positively correlated with each other $(r=0.56, p=0.013)$. Independent relationships between changes of f NEFA ( $r=-0.48, p=0.0035)$ and $\mathbf{g}$ IL-6 $(r=-0.44, p=0.056)$ with the relative improvement of HOMA-IR were also observed

was limited to only 19 individuals, all of whom were women, and that we were not able to study responses to a standardised meal. It was also not possible to assess insulin sensitivity by FSIVGTT or euglycaemic clamps in this study. 
The first important new finding was that the elevated fasting plasma glucagon concentrations in these severely obese participants were markedly reduced after RYGBP. This observation may be of physiological significance, as changes of fasting glucagon concentrations were significantly and independently associated with the changes of fasting insulin at 12 months after surgery. This relationship was present when proportional changes of glucagon and insulin were examined. In multivariate analysis, changes of glucagon concentrations were independently related to the improvements of HOMA-IR following RYGBP. Previous studies of fasting glucagon concentrations before and after RYGBP have reported no change either at 12 weeks [21] or 6 to 9 months after surgery [22]. This discrepancy may be due to the limited time course of the former study; and in the second study, a closer examination of the data actually suggests a $28 \%$ reduction of 'pancreatic' glucagon levels in the six patients in which both pre- and post-surgical samples were measured.

Glucagon plays a key physiological role in the maintenance of fasting and postprandial glucose concentrations by stimulating hepatic glucose production (reviewed in Dunning and Gerich [23]). Inappropriately elevated plasma glucagon concentrations have been reported in humans with diabetes [24]. Future studies employing more direct methods for determining insulin sensitivity and hepatic glucose production will be required to determine the specific role of glucagon in the resolution of insulin resistance after RYGBP.

Although we did not have an opportunity to examine meal-induced gastrointestinal hormone responses in the present study, there is a growing consensus that changes in their secretion are likely to contribute to improvement of insulin sensitivity after RYGBP, and may do so prior to significant weight loss [25]. For example, postprandial GLP-1 levels increase as early as 2 days after RYGBP [26]; and in addition to its potential effects to stimulate insulin secretion, GLP-1 has actions to suppress endogenous glucose production, promote glucose uptake, and importantly, to reduce glucagon secretion [27]. Interestingly, our results suggest that the decrease in fasting glucagon concentrations may be specific for RYGBP, as another group has reported that they did not change in 13 patients who lost $25 \%$ of body weight after vertical gastric banding or jejunoileal bypass [18]. We did measure fasting total GLP-1 concentrations in the present study; however, no significant changes were observed (data not shown). Nonetheless, it is likely that postprandial GLP-1 secretion was increased, and it is possible that repeated intermittent increases of GLP-1 in the postprandial period contributed to a sustained decrease of alpha cell function and reduced pancreatic glucagon release.
Reduced fasting PP concentrations were also observed after RYGBP. While this has been reported in one study [28], another reported no change of PP after surgery [22]. This discrepancy may be due to small sample sizes or differences in surgical technique. PP is released from pancreatic $\mathrm{F}$ cells in response to food intake, gastrointestinal hormones and neuropeptides. Its most important regulator, however, is cholinergic vagal stimulation. Consequently, plasma PP concentrations are useful as a marker of pancreatic parasympathetic activity [29]. Our results suggest that this declines shortly after RYGBP, consistent with reductions of insulin and glucagon, since the secretion of these hormones is also regulated by the parasympathetic input to the islet [30]. These results suggest at least a partial loss of the vagal input to the pancreas following RYGBP.

The second major finding was that RYGBP reduced concentrations of most of the adipocyte hormones and inflammatory molecules studied: leptin, hsCRP, ASP, IL-6 and SICAM-1.

Visfatin is a secreted protein produced at high levels in visceral fat, and its plasma concentration is correlated with visceral adiposity [6]. Accordingly, plasma concentrations of visfatin are higher in humans with metabolic syndrome than in normal weight control individuals [31]. Visfatin was initially reported to have insulin-mimetic and adipogenic actions [6], but these results were not supported by a follow-up study [32]. However, in addition to reduced circulating visfatin concentrations, female mice heterozygous for visfatin deficiency exhibit impaired glucose tolerance, and islets isolated from these mice have reduced glucose-stimulated insulin secretion [32]. To date, studies examining changes in visfatin with weight loss have reported conflicting results: one study reported decreased visfatin following RYGBP [33], while a subsequent study reported an increase [34]. These discrepancies may be partially explained by the use of a C-terminal assay for visfatin that has recently been shown to lack specificity [35]. Employing an ELISA that instead detects full-length visfatin [35], we found: (1) visfatin concentrations were positively associated with body weight and waist circumference prior to surgery; (2) visfatin decreased progressively after RYGBP; and (3) reductions of visfatin were independently associated with the changes of fasting insulin concentrations and HOMA-IR (Fig. 3d and Table 3). These results are consistent with the hypothesis that visfatin is over-produced in severe obesity, possibly to compensate for insulin resistance by either increasing adipose mass or augmenting glucose-stimulated insulin secretion. It follows that the need for such a mechanism would be reduced in the weight-reduced state.

The role of the pro-inflammatory cytokine IL-6 in the insulin resistance associated with human obesity remains a subject of debate, as it has been reported to exert some 
insulin-sensitising effects in both healthy human individuals and those with type 2 diabetes [36]. Nonetheless, circulating IL-6 concentrations have been reported to be two- to fourfold higher in obese, type 2 diabetic patients than in normal weight individuals [37] and to decrease with weight loss [38]. In the present study, there were strong positive correlations between circulating IL-6 concentrations and measures of body adiposity prior to RYGBP. Furthermore, IL- 6 concentrations were reduced by $43 \pm 7 \%$ 12 months after surgery. At this time, IL-6 concentrations were positively correlated with central adiposity, fasting insulin and HOMA-IR. Last, there was a trend $(p=0.056)$ for a relationship between the changes of IL- 6 and the relative improvement in HOMA-IR, independent of age and changes of NEFA and HMW adiponectin (Table 3 and Fig. 3g). As proposed previously [38], in obesity, adipose tissue is likely to be a major contributor to elevated IL-6 concentrations, and the underlying stimulus for its production is removed by weight loss. In the weight-reduced state, subsequently, the relationship between IL-6 concentrations and insulin resistance is re-established.

Changes in RBP4 were also examined in the present study, at 1 and 12 months after RYGBP. Serum RBP4 concentrations are elevated in overweight/obese humans and positively correlated with BMI and insulin resistance [39]. In the present study, however, we found no evidence linking RBP4 with RYGBP-induced improvements in insulin sensitivity. Although RBP4 concentrations decreased by nearly 30\% 1 month after surgery, at 12 months there was no significant decrease relative to pre-surgical levels, despite marked weight loss and improved insulin resistance.

Studies of RBP4 concentrations during weight loss have shown significant reductions occur with weight loss of $>5 \%$. One study [40] reported that serum RBP4 concentrations in severely obese humans decreased by $\sim 26 \%$ at 6 months after gastric banding, when participants had lost $13 \%$ of their initial weight. In patients undergoing vertical banded gastroplasty, a similar reduction of RBP4 concentrations ( $20 \%$ after 1 month) was reported, although it was not stated how much weight the patients lost [41]. In healthy, insulin-sensitive menopausal women, however, weight loss of $5 \%$ over 13 weeks, achieved by exercise and reduction of energy intake by $2,510 \mathrm{~kJ} /$ day $(600 \mathrm{kcal} /$ day), did not change serum RBP4 concentrations, despite a $20 \%$ decrease of fasting insulin [42]. We also measured RBP4 concentrations in 14 healthy women before and after a 7 day period of energy restriction to $2,635 \mathrm{~kJ} /$ day (630 kcal/day) [43], during which the women lost $4 \%$ of their body weight and plasma insulin concentrations decreased by $46 \%$. There was no significant change of fasting RBP4 concentrations (before, $46.2 \pm 5.9 \mathrm{mg} / \mathrm{l}$; after, $40.9 \pm 4.9 \mathrm{mg} / \mathrm{l} ; p=0.33)$.
Moreover, there is some evidence to suggest that weight loss-mediated reductions in RBP4 concentrations may not be sustained in the long-term (12 months). Similar results to ours have been reported recently in a study of obese women consuming a very low energy diet $(3,350 \mathrm{~kJ}$ [800 kcal/day]) for 4 weeks: a $\sim 7 \%$ reduction in body weight was associated with a $15-20 \%$ decrease in plasma RBP4 concentrations [44]. Interestingly, an additional 3\% weight loss in these patients, achieved by a low-energy diet for 8 weeks followed by a weight-maintaining diet for $12-$ 16 weeks, resulted in a modest increase of RBP4 concentrations, although they were still reduced compared with initial levels. This and other evidence [45] suggests that changes in RBP4 concentrations may be more indicative of energy intake than changes in body weight per se. It should also be noted that adipose tissue is not the sole source of RBP4, as it is most highly produced in liver, with adipose tissue levels being about $20 \%$ of hepatic levels [46].

Last, as we previously reported for total adiponectin concentrations [7], pre-surgical concentrations of HMW adiponectin independently predicted weight loss following RYGBP, with higher concentrations associated with greater weight loss. Pre-operative HMW adiponectin concentrations also predicted loss of fat mass. Adiponectin stimulates fatty acid oxidation in liver and skeletal muscle [47], so it is reasonable to hypothesise that a higher initial concentration could enhance fat oxidation during negative energy balance; however, this hypothesis will need to be addressed in future studies.

The present study was designed to be a comprehensive investigation of the longitudinal endocrine and metabolic changes following RYGBP in severely obese women, with an emphasis on pancreatic and adipocyte hormones and markers of inflammation. While the contribution of gastrointestinal hormones to improvements of insulin resistance after RYGBP has been more extensively studied, the present findings suggest that hormones from the pancreas (glucagon and PP in particular) and adipose tissue (visfatin and HMW adiponectin) may contribute to the resolution of insulin resistance in the long term. Future studies, involving larger numbers of patients of both sexes as well as mechanistic studies, will be required to fully determine the contributions of each of these hormones. Accordingly, appropriate enhancement or blocking of the effects of specific hormones may lead to the development of new, non-surgical approaches for medical management of the co-morbidities associated with severe obesity.

Acknowledgements We acknowledge the expert technical assistance of J. Graham and E. Nuñez. This study was supported by a grant award from the University of California Davis Health Care Systems. It was also supported by the UC Davis Clinical and Translational Science Center (grant number UL1 RR024146) and from the National Center for Research Resources (NCRR), a component of the National 
Institutes of Health (NIH) and NIH Roadmap for Medical Research. The content of this manuscript is solely the responsibility of the authors and does not necessarily represent the official view of NCRR or NIH. P. J. Havel's laboratory receives research support from NIH grants: HL-075675, AT-002599, AT-00293, and the American Diabetes Association. M. M. Swarbrick is supported by a New Investigator Award from NAASO, The Obesity Society.

Duality of interest The authors declare that there is no duality of interest associated with this manuscript.

\section{References}

1. Buchwald H, Avidor Y, Braunwald E et al (2004) Bariatric surgery: a systematic review and meta-analysis. JAMA 292:17241737

2. Zhao Y, Encinosa W (2007) Agency for Healthcare Research and Quality. Bariatric surgery utilization and outcomes in 1998 and 2004. Statistical Brief \#23. Available from http://www.hcup-us. ahrq.gov/reports/statbriefs/sb23.pdf, accessed 15 July 2008

3. Buchwald H, Williams SE (2004) Bariatric surgery worldwide 2003. Obes Surg 14:1157-1164

4. le Roux CW, Bloom SR (2005) Why do patients lose weight after Roux-en-Y gastric bypass. J Clin Endocrinol Metab 90:591-592

5. Havel PJ (2004) Update on adipocyte hormones: regulation of energy balance and carbohydrate/lipid metabolism. Diabetes 53 (Suppl 1):S143-S151

6. Fukuhara A, Matsuda M, Nishizawa M et al (2005) Visfatin: a protein secreted by visceral fat that mimics the effects of insulin. Science 307:426-430

7. Faraj M, Havel PJ, Phelis S, Blank D, Sniderman AD, Cianflone K (2003) Plasma acylation-stimulating protein, adiponectin, leptin, and ghrelin before and after weight loss induced by gastric bypass surgery in morbidly obese subjects. J Clin Endocrinol Metab 88:1594-1602

8. Yang Q, Graham TE, Mody N et al (2005) Serum retinol binding protein 4 contributes to insulin resistance in obesity and type 2 diabetes. Nature 436:356-362

9. Weisberg SP, McCann D, Desai M, Rosenbaum M, Leibel RL, Ferrante AW Jr (2003) Obesity is associated with macrophage accumulation in adipose tissue. J Clin Invest 112:1796-1808

10. Hak AE, Stehouwer CD, Bots ML et al (1999) Associations of Creactive protein with measures of obesity, insulin resistance, and subclinical atherosclerosis in healthy, middle-aged women. Arterioscler Thromb Vasc Biol 19:1986-1991

11. Kern PA, Ranganathan S, Li C, Wood L, Ranganathan G (2001) Adipose tissue tumor necrosis factor and interleukin-6 expression in human obesity and insulin resistance. Am J Physiol Endocrinol Metab 280:E745-E751

12. Kim CS, Park HS, Kawada T et al (2006) Circulating levels of MCP-1 and IL-8 are elevated in human obese subjects and associated with obesity-related parameters. Int $\mathrm{J}$ Obes (Lond) 30:1347-1355

13. Ferri C, Desideri G, Valenti M et al (1999) Early upregulation of endothelial adhesion molecules in obese hypertensive men. Hypertension 34:568-573

14. Van Gaal LF, Mertens IL, De Block CE (2006) Mechanisms linking obesity with cardiovascular disease. Nature 444:875-880

15. Swarbrick MM, Austrheim-Smith IT, Stanhope KL et al (2006) Circulating concentrations of high-molecular-weight adiponectin are increased following Roux-en-Y gastric bypass surgery. Diabetologia 49:2552-2558
16. Matthews DR, Hosker JP, Rudenski AS, Naylor BA, Treacher DF, Turner RC (1985) Homeostasis model assessment: insulin resistance and beta-cell function from fasting plasma glucose and insulin concentrations in man. Diabetologia 28:412-419

17. Saad MF, Kahn SE, Nelson RG et al (1990) Disproportionately elevated proinsulin in Pima Indians with noninsulin-dependent diabetes mellitus. J Clin Endocrinol Metab 70:1247-1253

18. Guldstrand M, Ahren B, Adamson U (2003) Improved beta-cell function after standardized weight reduction in severely obese subjects. Am J Physiol Endocrinol Metab 284:E557-E565

19. Havel PJ, Ahren B (1997) Activation of autonomic nerves and the adrenal medulla contributes to increased glucagon secretion during moderate insulin-induced hypoglycemia in women. Diabetes $46: 801-807$

20. Havel PJ, Townsend R, Chaump L, Teff K (1999) High-fat meals reduce 24 -h circulating leptin concentrations in women. Diabetes 48:334-341

21. Clements RH, Gonzalez QH, Long CI, Wittert G, Laws HL (2004) Hormonal changes after Roux-en Y gastric bypass for morbid obesity and the control of type-II diabetes mellitus. Am Surg 70:1-4 (discussion 4-5)

22. Meryn S, Stein D, Straus EW (1986) Fasting- and meal-stimulated peptide hormone concentrations before and after gastric surgery for morbid obesity. Metabolism 35:798-802

23. Dunning BE, Gerich JE (2007) The role of alpha-cell dysregulation in fasting and postprandial hyperglycemia in type 2 diabetes and therapeutic implications. Endocr Rev 28:253-283

24. Gastaldelli A, Baldi S, Pettiti M et al (2000) Influence of obesity and type 2 diabetes on gluconeogenesis and glucose output in humans: a quantitative study. Diabetes 49:1367-1373

25. Cummings DE, Overduin J, Foster-Schubert KE (2004) Gastric bypass for obesity: mechanisms of weight loss and diabetes resolution. J Clin Endocrinol Metab 89:2608-2615

26. le Roux CW, Welbourn R, Werling M et al (2007) Gut hormones as mediators of appetite and weight loss after Roux-en-Y gastric bypass. Ann Surg 246:780-785

27. Salehi M, Aulinger BA, D’Alessio DA (2008) Targeting b-cell mass in type 2 diabetes: promise and limitations of new drugs based on incretins. Endocr Rev 29:367-379 doi:10.1210/er.20070031

28. Schrumpf E, Linnestad P, Nygaard K, Giercksky KE, Fausa O (1981) Pancreatic polypeptide secretion before and after gastric bypass surgery for morbid obesity. Scand J Gastroenterol 16:1009-1014

29. Schwartz TW (1983) Pancreatic polypeptide: a unique model for vagal control of endocrine systems. J Auton Nerv Syst 9:99-111

30. Havel PJ, Taborsky GJ Jr (1989) The contribution of the autonomic nervous system to changes of glucagon and insulin secretion during hypoglycemic stress. Endocr Rev 10:332-350

31. Filippatos TD, Derdemezis CS, Kiortsis DN, Tselepis AD, Elisaf MS (2007) Increased plasma levels of visfatin/pre-B cell colonyenhancing factor in obese and overweight patients with metabolic syndrome. J Endocrinol Invest 30:323-326

32. Revollo JR, Korner A, Mills KF et al (2007) Nampt/PBEF/ Visfatin regulates insulin secretion in beta cells as a systemic NAD biosynthetic enzyme. Cell Metab 6:363-375

33. Manco M, Fernandez-Real JM, Equitani F et al (2007) Effect of massive weight loss on inflammatory adipocytokines and the innate immune system in morbidly obese women. J Clin Endocrinol Metab 92:483-490

34. Krzyzanowska K, Mittermayer F, Krugluger W, Kopp HP, Schernthaner G (2006) Increase in visfatin after weight loss induced by gastroplastic surgery. Obesity (Silver Spring) 14:1886-1889

35. Korner A, Garten A, Bluher M, Tauscher R, Kratzsch J, Kiess W (2007) Molecular characteristics of serum visfatin and differential 
detection by immunoassays. J Clin Endocrinol Metab 92:47834791

36. Pedersen BK, Febbraio MA (2007) Point: interleukin-6 does have a beneficial role in insulin sensitivity and glucose homeostasis. J Appl Physiol 102:814-816

37. Vozarova B, Weyer C, Hanson K, Tataranni PA, Bogardus C, Pratley RE (2001) Circulating interleukin-6 in relation to adiposity, insulin action, and insulin secretion. Obes Res 9:414-417

38. Bastard JP, Jardel C, Bruckert E et al (2000) Elevated levels of interleukin 6 are reduced in serum and subcutaneous adipose tissue of obese women after weight loss. J Clin Endocrinol Metab 85:3338-3342

39. Graham TE, Yang Q, Bluher $M$ et al (2006) Retinol-binding protein 4 and insulin resistance in lean, obese, and diabetic subjects. N Engl J Med 354:2552-2563

40. Haider DG, Schindler K, Prager G et al (2007) Serum retinolbinding protein 4 is reduced after weight loss in morbidly obese subjects. J Clin Endocrinol Metab 92:1168-1171

41. Berggren Soderlund M, Fex G, Nilsson-Ehle P (2003) Decreasing serum concentrations of all-trans, 13-cis retinoic acids and retinol during fasting and caloric restriction. J Intern Med 253:375-380
42. Janke J, Engeli S, Boschmann M et al (2006) Retinol-binding protein 4 in human obesity. Diabetes 55:2805-2810

43. Dubuc GR, Phinney SD, Stern JS, Havel PJ (1998) Changes of serum leptin and endocrine and metabolic parameters after 7 days of energy restriction in men and women. Metabolism 47:429-434

44. Vitkova M, Klimcakova E, Kovacikova M et al (2007) Plasma levels and adipose tissue messenger ribonucleic acid expression of retinol-binding protein 4 are reduced during calorie restriction in obese subjects but are not related to diet-induced changes in insulin sensitivity. J Clin Endocrinol Metab 92:2330-2335

45. Shetty PS, Watrasiewicz KE, Jung RT, James WP (1979) Rapidturnover transport proteins: an index of subclinical protein-energy malnutrition. Lancet 2:230-232

46. Tsutsumi C, Okuno M, Tannous L et al (1992) Retinoids and retinoid-binding protein expression in rat adipocytes. J Biol Chem 267:1805-1810

47. Yamauchi T, Kamon J, Minokoshi Y et al (2002) Adiponectin stimulates glucose utilization and fatty-acid oxidation by activating AMP-activated protein kinase. Nat Med 8:1288-1295 